\title{
Bone strength beyond bone mass
}

\section{The Galle Medical Association Oration 2019}

\author{
Alwis G \\ Department of Anatomy, Faculty of Medicine, University of Ruhuna, Galle, Sri Lanka. \\ Correspondence: Dr. Gayani Alwis \\ e-mail: galwis@med.ruh.ac.lk \\ (D) https://orcid.org/0000-0002-4025-9709
}

\section{Introduction}

Fragility hip fracture, a serious manifestation of osteoporosis in the elderly, is a worldwide problem associated with low bone strength. With the increasing elderly population, incidence of hip fracture is estimated to be increased - especially in Asian countries (1). Low bone mass has been recognised as the strongest risk factor for fragility fracture. However, studies have reported the limitations of assessing bone strength or fracture risk based on bone mass measurements alone $(2,3)$. The ability of a bone to resist fracture, or whole bone strength, depends not only on the bone mass, but also on bone size, shape, spatial distribution of bone mass, bone geometry, cortical and trabecular microarchitecture and the balance between bone formation and bone resorption (4). Therefore, the study of factors associated with geometric adaptations and biomechanical behaviours of the hip, as a compensatory mechanism for enhancing bone strength against low bone mass and hip fracture risk is important.

Bone undergoes formation and resorption that are coupled processess and during growing ages bone formation predominates, leading to an increase in bone mass till it reaches the peak bone mass (PBM). PBM is an important determinant of bone strength , aiming fracture prevention in old age because $50 \%$ of bone mass at age 70 is estimated to be predicted by PBM (5). Theoretical analyses further estimate that a 10\% increase in PBM could delay the development of osteoporosis by 13 years. Physical activity (PA) is recognised as one of the strategies to optimize skeletal development in growing children.
The pre- and early peri-pubertal period, a period with fast skeletal growth, is considered as a window of opportunity to enhance bone strength by physical exercises (6). However, most exercise interventions in children have predominantly been focused on volunteers, with specifically designed high-impact exercise intervention programs or organised sports activities using competitive athletics (6). Therefore, when we plan to design an exercise program as a preventive strategy against low bone strength, it should come from a population-based level where every child could enjoy in a safe environment. With sophisticated lifestyles and the development of modern technology, physical inactivities and sedentary lifestyle have become increasingly prevelant in modern societes. As a result, noncommunicable diseases and childhood obesity have been recognised as growing global health concerns. Meanwhile children and adolescents in Sri Lanka have also moved away from the playground, or active lifestyle. Physical inactivity is identified as one of the most prominent risk factors for both low bone strength and childhood obesity.

Anthropometric parameters such as height, weight and body mass index (BMI) are important practising growth indicators to assess the adequacy of growth. The Pediatric Official Positions of the International Society for Clinical Densitometry (ISCD) 2007 revealed the requirement of age and sex specific normative data in children and adolescents including the anthropometry, body composition, and bone measurements of multiple skeletal regions along with their relationships when evaluating growth disturbances (7). 
Among the development of different bones, the development of femoral neck (FN) geometry is of crucial importance to increase bone strength as a fracture prevention strategy in elderly. However, the lack of published data regarding age and sex specific geometric adaptations at hip emphasizes the importance of studying FN geometry. FN connects the femoral head to the shaft. The narrowest FN represents the smallest periosteal diameter (PD) and is identified as the weakest point. This narrowest neck is used as the region of interest when determining the geometric indices and biomechanical measures of the FN. Using the facts described above, Thomas. J. Beck, an American scientist, designed a cost effective dual energy X-ray absorptiometry (DXA) based advanced hip structural analysis (HSA) technique, with minimum radiation exposure, to estimate the geometry and biomechanical strength of the proximal femuor (8).

Based on this background, the study series presented in the oration was planned in two phases, and the overall aim of this work is to improve bone strength, aiming future fracture prevention.

\section{Phase I: Physical education exercise intervention}

- The main objective of this study was to evaluate whether a general school-curriculum based moderately intense physical education intervention, where every child could participate, leads to improved accrual of bone mass, bone size and FN structure in a group of pre-pubertal children.

\section{Phase II: Three cross-sectional studies}

- Study I evaluated the age and gender specific changes in the normative anthropometric, body composition and bone growth measurements in children and adolescents to evaluate the timing and magnitude of accrual of bone mineral and bone size in different skeletal regions.

- Study II evaluated the age and gender specific normative FN geometry and biomechanical estimates of FN and estimated whether changes in geometric adaptations and biomechanical behaviours at $\mathrm{FN}$ during growth counteracted the age related loss of bone mass in elderly as a fracture preventive strategy.
- Study III evaluated the relationships of anthropometry and body composition on FN geometry in children and adolescents.

\section{Methods}

In this oration, I wish to present the findings of a research project known as "paediatric osteoporosis prevention (POP) study", which was conducted in Malmö, Lund University, Sweden. In this study series, More than $99 \%$ of participants were Caucasians and none were on lifestyle restrictions or had diseases or medications known to affect growth or bone metabolism. An intervieweradministered questionnaire was used to evaluate life-style factors such as nutritional habits, level of PA, smoking, alcohol, and diseases and medications. The questionnaires of younger children were assessed together with the parents or guardians. Pubertal status was assessed using Tanner staging. All the studies were approved by the Ethics Committee of Lund University and the Radiographic Committee at Malmo University Hospital, Malmo, Sweden and were conducted according to the Helsinki Declaration. Informed written consent was obtained from parents or guardians of participants and the study participants before starting the study. Statistical calculations were performed using Statistica version Statistica ${ }^{\circledR}$, version 6.1 (StatWin ${ }^{\circledR}$ ) and a p-value of $<0.05$ was considered as a statistically significant difference.

\section{Phase I: Physical education intervention}

Study design and study participants: For the intervention study, four schools within similar socioeconomic backgrounds were selected. One school, which was geographically away from the other three, was invited to participate as the intervention school and the other three schools participated as control schools. The intervention included moderately intense physical activities within the general Swedish physical education (PE) school curriculum. For the intervention, just the duration of PE classes was increased without changing any activities within the school curriculum. The duration of PE was increased from 60 minutes or 1 - 2 lessons per week, to 200 minutes per week. That is, 40 minutes per each school day. The PE curriculum includes a variety of 
free activities, such as ball games, running, jumping and climbing under the supervision of the ordinary class teacher.

At baseline, 54 girls in the intervention and 64 in the control group participated. Forty-nine girls in the intervention and 50 in the control group participated at the two-year follow-up. A sample of 84 boys in the intervention and 68 in the control group participated at baseline. Eighty boys in the intervention and 57 in the control group participated at the two-year follow-up.

All measurements (level of PA, anthropometry and bone measurements) were taken at baseline, before the intervention, and at one year and at two-year follow-ups. In the PE intervention, absolute annual changes in bone mass and bone structure were calculated as changes per 365 days and were compared between the intervention and the control group at one year and two year follow-ups by student's t-test between means.

Anthropometric measurements: Body weight was measured with an electric scale to the nearest $0.1 \mathrm{~kg}$ and body height by a wall-tapered height meter to the nearest $0.5 \mathrm{~cm}$. BMI was calculated as weight in kilograms divided by height in meters squared.

Body composition and bone measurements: Fat mass and lean mass were evaluated from the total body (TB) scan in TB, arms and legs using DXA scan (DXA, DPX-L version 1.3z, Lunar ${ }^{\circledR}$, Madison, WI). Bone mineral content (BMC) and bone mineral density (BMD) were evaluated for the TB (including head), arms, legs and total spine by a TB scan; femoral neck (FN) by a hip scan; and third lumbar vertebra (L3) and second to fourth lumbar vertebrae (L2-L4) by a lumbar spine (LS) scan. The width of the L3 was estimated from the LS scans and the width of the FN was estimated from the hip scans. Pediatric software was used in children below $35 \mathrm{~kg}$ in weight and in all other participants, adult standard software was used. The measurements were done according to the standard procedure with the participants dressed in light clothes.

The following hip geometry and biomechanical indices, namely, FN width (FNW, cm), crosssectional area $\left(\mathrm{CSA}, \mathrm{cm}^{2}\right)$, cross-sectional moment of inertia (CSMI, $\left.\mathrm{cm}^{4}\right)$, section modulus $\left(\mathrm{Z}, \mathrm{cm}^{3}\right)$,
$\mathrm{BMC}$ and $\mathrm{BMD}$, at the narrowest $\mathrm{FN}$, were evaluated by HSA software (Lunar DPX-L 4.7E). In addition, endosteal diameter (ED, $\mathrm{cm})$ was estimated using the algorithm reported in the literature (9). Mean cortical thickness (CT, cm) was calculated as the difference between PD and ED, divided by two.

Assessment of the level of PA: Subjective level of PA and lifestyle factors were assessed using an interviewer administered questionnaire. Objective level of PA was assessed using the MTI accelerometer, model 7164 (Manufacturing Technology Incorporated, Fort Walton Beach, FL, USA).

Results of the exercise intervention: At baseline, in both girls and boys, the two groups did not differ with regard to lifestyle factors, age, anthropometrics or bone parameters. When the annual changes were compared, both boys and girls in the intervention group had significantly higher accrual of BMC and larger gain in bone size in the lumbar vertebrae. No differences between intervention and control groups were observed for annual changes in the FN bone mineral accrual or FN geometry and biomechanical changes measured at one year and two-year follow-up. The positive effects in the lumbar spine were less in boys than in girls (10). All skeletal responses compared to controls in percentage gain at one year and two year follow-ups are summarised in Table 1.

The larger proportion of trabecular bone in lumbar vertebrae than in FN could partly explain the discrepancy, as skeletal response to mechanical load is more marked in trabecular regions than in cortical regions due to the larger surface-to-volume ratio in trabecular bone. In addition, girls of the same chronological age are generally closer to puberty than boys of the same age. It is also widely accepted that the skeleton produces a greater response to mechanical load in the early pubertal period. Because of the earlier onset of puberty, girls reach peak BMC velocity roughly 1.5 years earlier than boys. 
The findings that LS bone mass increases initially more in girls than in boys of the same chronological age is consistent with literature from children and adolescents from a similar background. The finding is of considerable interest as high-intensity activities with few daily repetitions are more important than a long duration of exercise if the purpose is to reach skeletal benefits (5). Accelerometer data further revealed that all of these children were physically active and reached the international recommended level of PA, which is more than 60 minutes of MVPA per day (Table 2).
Based on these findings the following conclusions were made. The increased duration of physical activity within the school PE curriculum was associated with beneficial accrual of bone mass and bone size in the LS but not in the FN. All the children in this study were physically active. None significant results at $\mathrm{FN}$ need to be studied in future studies. The findings of this study were published in SCI cited journals (11-14).

Table 1: The summary of skeletal responses compared to controls (percentage) at one year and two year follow-ups. $* \mathrm{P}<0.05, * * \mathrm{P}<0.01, * * * \mathrm{P}<0.001$ and not significant (NS)

\begin{tabular}{|c|c|c|c|c|c|c|}
\hline & $\begin{array}{l}\text { L2-L4 } \\
\text { BMC }\end{array}$ & $\begin{array}{l}\text { L2-L4 } \\
\text { BMD }\end{array}$ & $\begin{array}{c}\text { L3 } \\
\text { BMC }\end{array}$ & $\begin{array}{c}\text { L3 } \\
\text { BMD }\end{array}$ & $\begin{array}{c}\text { L3 } \\
\text { Width }\end{array}$ & $\begin{array}{c}\text { Femoral neck } \\
\text { BMC, BMD, width, } \\
\text { CSMI, CSA, Z }\end{array}$ \\
\hline Girls - 1 year & $4.7 * * *$ & $2.8 * * *$ & $9.5 * * *$ & $3.1 * * *$ & $2.9 * * *$ & NS \\
\hline Girls - 2 year & $3.8^{* *}$ & $1.2^{*}$ & $7.2 * * *$ & $1.6^{*}$ & $1.8^{* * *}$ & NS \\
\hline Boys - 1 year & $3.2 * *$ & $1.9^{*}$ & $5.2 * * *$ & $2.1^{*}$ & $2.3 * *$ & NS \\
\hline Boys - 2 year & NS & NS & $3.0 * *$ & NS & $1.3 * *$ & NS \\
\hline
\end{tabular}

Table 2: Level of physical activity evaluated by accelerometers $* * \mathrm{p}<0.01, * * * \mathrm{p}<0.001$

\begin{tabular}{lccccccc}
\hline At follow-up & $\begin{array}{c}\text { Intervention } \\
\text { Girls N=41 }\end{array}$ & $\begin{array}{c}\text { Controls } \\
\text { Girls N=40 }\end{array}$ & $\begin{array}{c}\text { Intervention } \\
\text { Boys N=72 }\end{array}$ & $\begin{array}{c}\text { Controls } \\
\text { Boys } \\
\text { N=55 }\end{array}$ \\
\hline Recording time (hrs/day) & $12(1)$ & 12 & $(1)$ & 12 & $(1)$ & 13 & $(1)$ \\
Mean activity & $644(184)$ & $590(115)$ & $770(267)$ & $728(211)$ \\
(counts/minute/day) & $194(45)$ & 185 & $(35)$ & 211 & $(55)$ & 209 & $(45)$ \\
$>3$ METS (minutes/day) & $34(15)$ & 35 & $(12)$ & 44 & $(21)$ & 48 & $(19)$ \\
$>6$ METS (minutes/day) & 13 & $(8)$ & 11 & $(6)$ & $16(10)$ & 15 & $(9)$ \\
$>6000$ counts/min (min/day) & $3^{* * *}(3)$ & 1 & $(1)$ & $4^{* *}(3)$ & 2 & $(3)$ \\
$>10000$ counts/min (min/day) & & & & & & &
\end{tabular}




\section{DXA derived normative data in children and adolescents:}

This cross-sectional study was designed to explore the findings of the PE intervention study and to fulfill the requirement of normative data to assess growth in normal children and adolescents (7). For this study, 710 females with a mean age of 13.2 years (range $5.9-30.3$ ), and 759 males with a mean age 13.2 years (range 6.4 - 30.4), were included. Participants below the age of 18 were randomly selected from 10 schools in the city of Malmö and adult participants were recruited from surrounding communities. Height and weight were measured according to the standard protocols and BMI was calculated. Using DXA technique, lean mass, fat mass, $\mathrm{BMC}$, and $\mathrm{BMD}$ were measured at $\mathrm{TB}$, arms and legs. BMC, BMD, bone size and bone area were measured at LS and FN. Age and sex specific normative data during growth were plotted in one-year age groups as mean (SE).

\section{Results}

\section{Anthropometry, muscle mass, fat mass and bone mass:}

During pre-pubertal years, gain in height, weight, and TB bone mass is linear and the rate of accrual is similar in girls and boys. When looking at the gain in TB and appendicular muscle mass, boys showed higher muscle mass from the beginning, and with the pubertal growth spurt, sex differences became more apparent. However, the gain in TB and appendicular fat mass showed a different pattern, with girls in general having higher values compared to boys (Figure 1). Further, when looking at the accrual of bone mass and bone size in LS, girls reach peak velocity of BMC and BMD in LS roughly $1.5-2.0$ years earlier than boys do, in that bone mass accrual in LS increases initially more in girls than in boys of the same chronological age (Figure 2 ). This could be due to the earlier onset of puberty in girls. This finding clarifies the exercise induced higher gain in LS bone mass and bone size of girls in the intervention group.

Compared to other skeletal sites, BMC and BMD accrual at $\mathrm{FN}$ showed a different pattern in both girls and boys. There is a tendency for an early peak and decline in FN bone mass already at the end of the second decade compared to the stable or slight further increase in TB BMD and LS BMD, (Figure 2 ), being in accordance with previous reports in Swedish adolescents (15). However, the lower value of FN BMD in girls at all ages compared to boys is also consistent with the literature in Canadian children. In addition, the early onset of bone mass loss in FN compared to LS is also consistance with previous reports (16).

In conclusion, except in LS, adolescent boys showed a higher bone mass, bone size and muscle mass whereas girls showed higher fat mass throughout the measured age range. FN showed a different growth pattern with early peak and decline of bone mass compared to the other skeletal sites. These findings need to be further studied in future longitudinal studies. The present study supports the view of a heterogeneous muscular skeletal development during growth. Findings of this study were published in ACTA paediatrica in 2010 (17).

\section{Femoral neck bone strength estimated by HSA:}

In our previous published study by Alwis et.al, (17), FN showed different age and sex specific growth patterns compared to the other skeletal sites as detected by conventional DXA. Therefore, this study was aimed to evaluate age and sex specific changes in normative FN geometry and biomechanical estimates, and to evaluate whether these changes in FN could compensate for the age related loss of bone mass to maintain FN bone strength. This study was based on HSA measurements of 1760 study participants aged 6-90 years; FN geometry data from age 6 to 19 years were presented in one-year age groups, and from age 20 to 90 years were presented in 10-year age groups.

\section{FN geometric adaptations in children, adolescents and adults:}

When plotting the age specific changes in $\mathrm{FN}$ $\mathrm{BMC}, \mathrm{BMD}, \mathrm{PD}$, and ED the curves increased with higher ages until statistically significant break points were reached at age 17 in girls and 19 in boys. A similar pattern was observed in CT, CSA, CSMI and $Z$ (Figure 3). In adulthood, after the break points, while BMC and BMD have decreased, PD and ED 
have increased with advancing ages. This showed the distribution of bone mass further away from the central axis of FN (Figure 3 ).

In adults, as shown in second half of the table 3, the relative decrease in BMC, BMD, CT, CSA, CSMI and $\mathrm{Z}$ from peak to old age was more marked

A

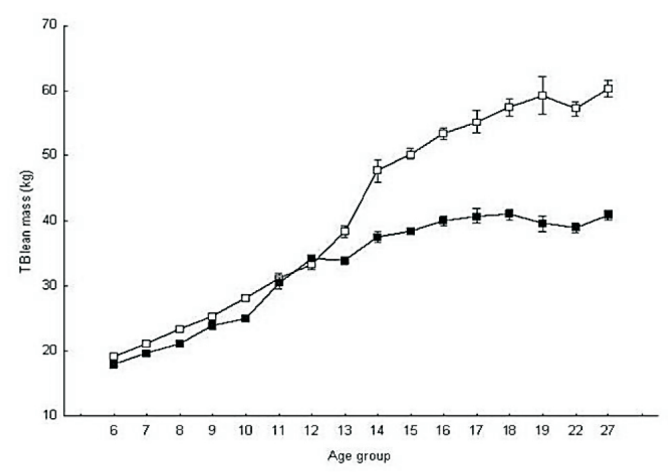

C

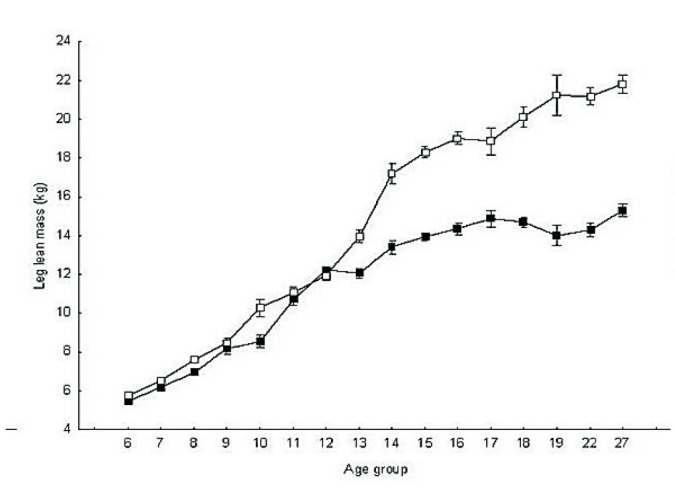

$\mathbf{E}$

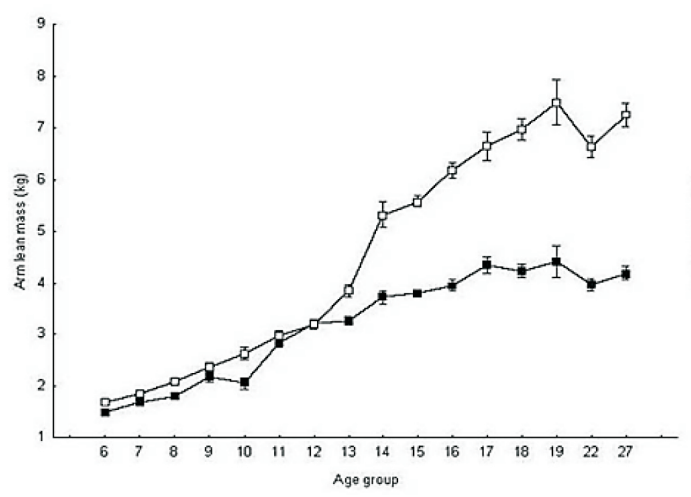

in women than in men, whereas the relative expansion in ED and PD from peak to old age was more marked in men than in women (Table 3). The expansion in ED was greater than the expansion in $\mathrm{PD}$, resulting in a diminishing $\mathrm{CT}$ in both sexes, which is more marked in women than in men (9).
B

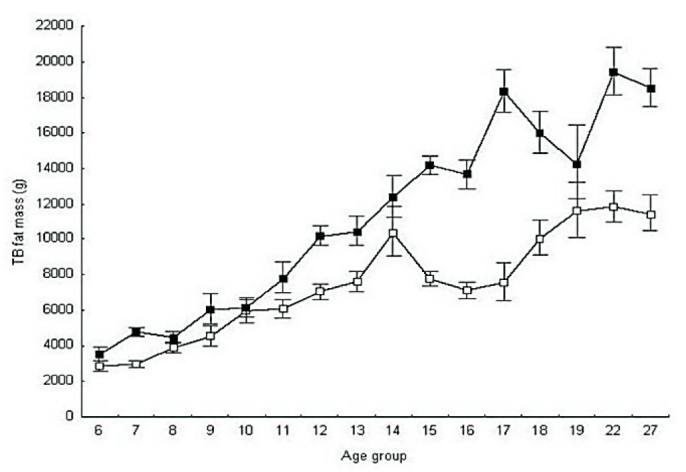

D

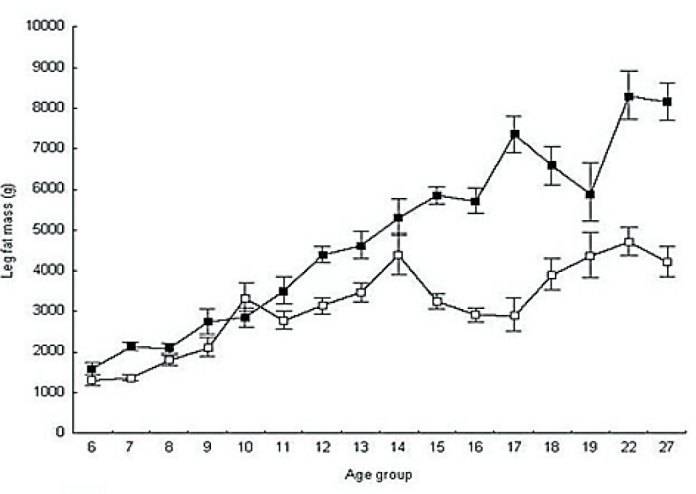

F

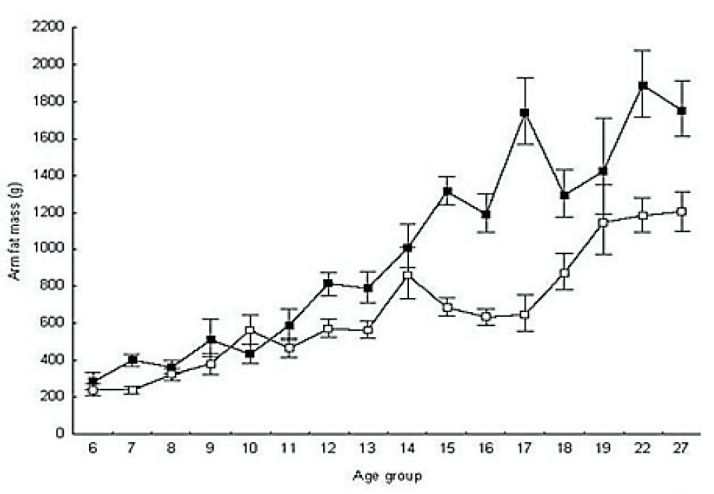

Boys

Figure 1: Age and sex specific changes in lean mass and fat mass regions and FN width A- total body lean mass, B- total body fat madd, C- leg lean mass, D- leg fat mass, E- arm lean mass and F- arm fat mass. Data presented as mean (SE) 
A

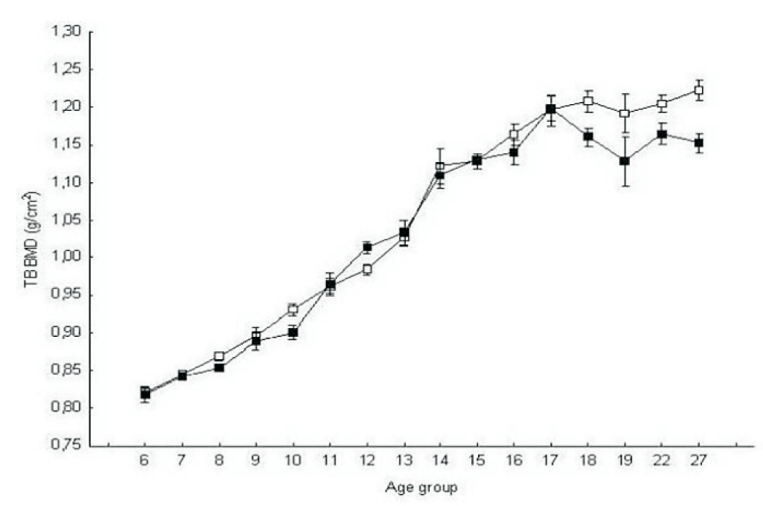

C

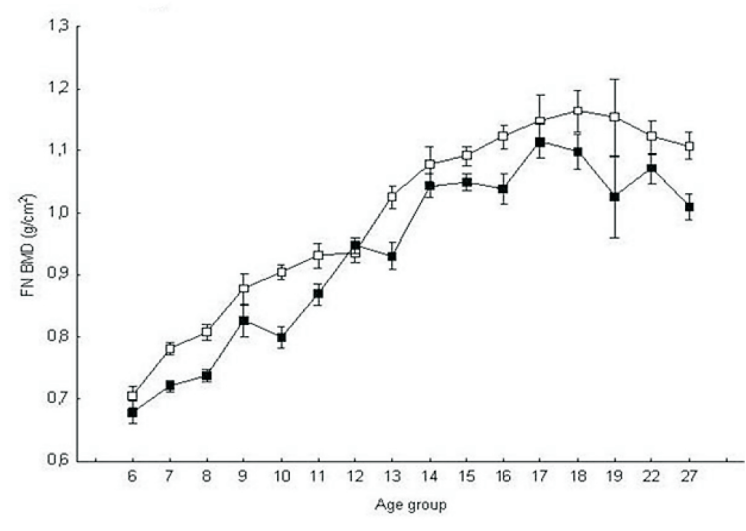

E

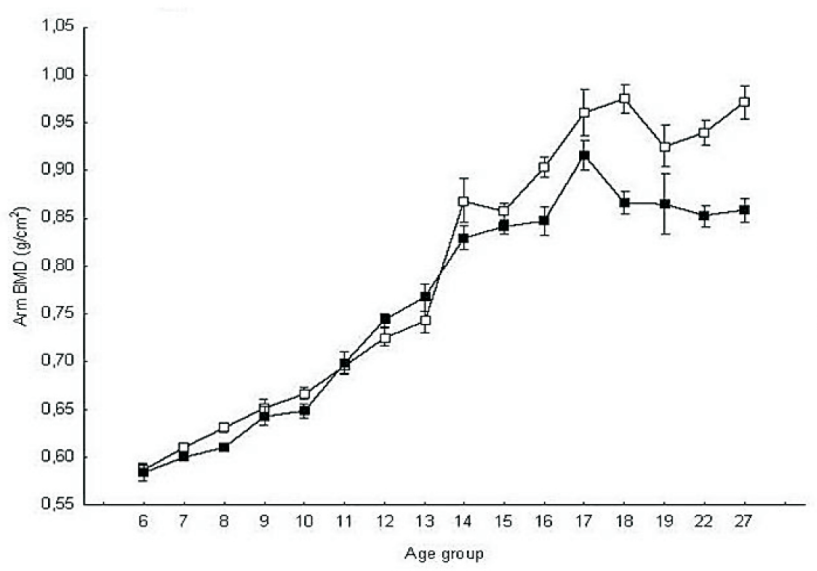

B

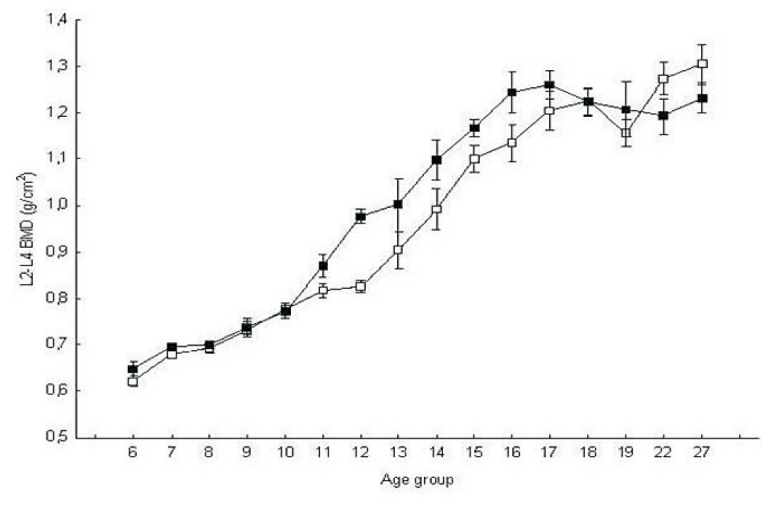

D

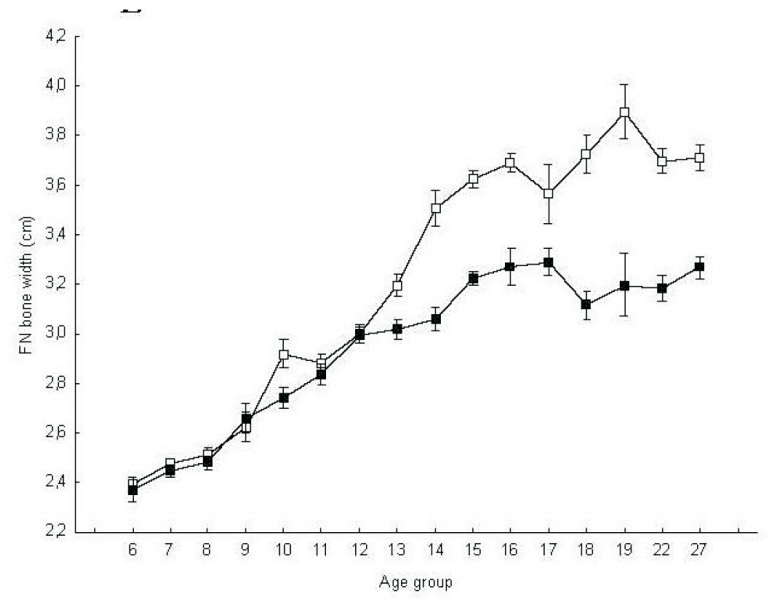

$\mathbf{F}$

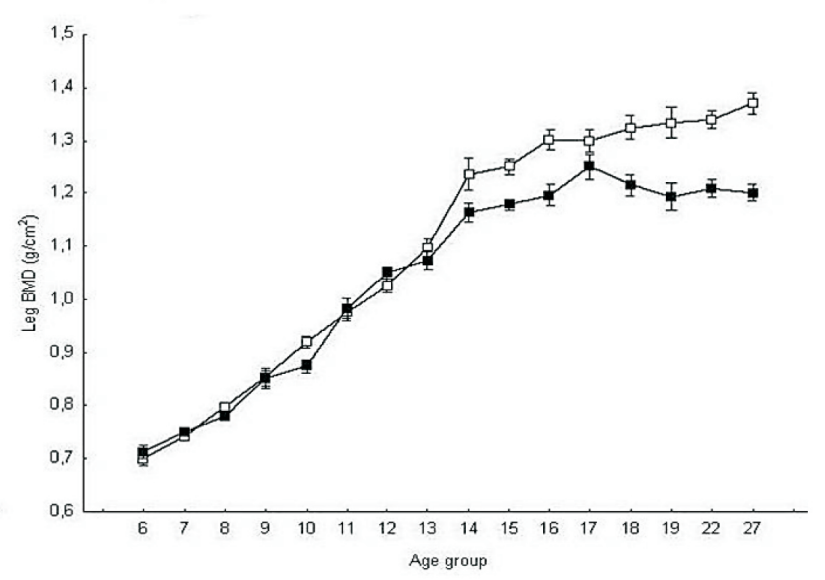

Boys

Figure 2: Age and sex specific changes in BMD in different skeletal regions and FN width A- total body, B- lumbar spine, C- femoral neck, DF- FN width, E-arm and F- leg. Data presented as mean (SE) 
A

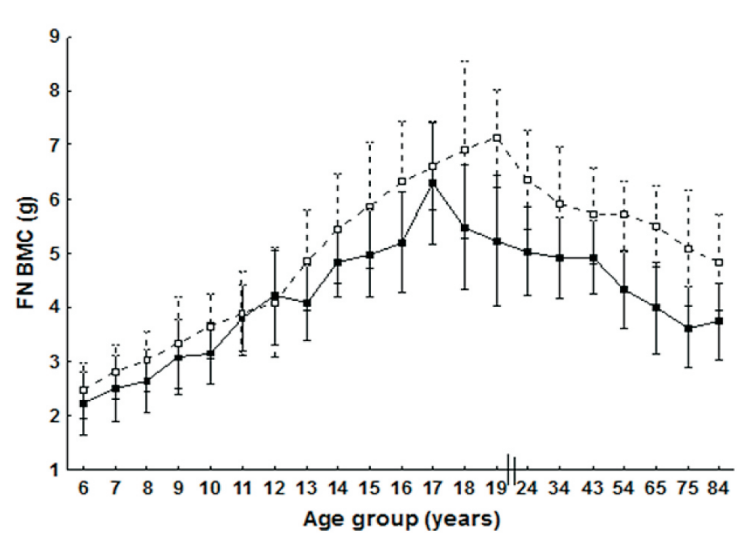

$\mathrm{C}$

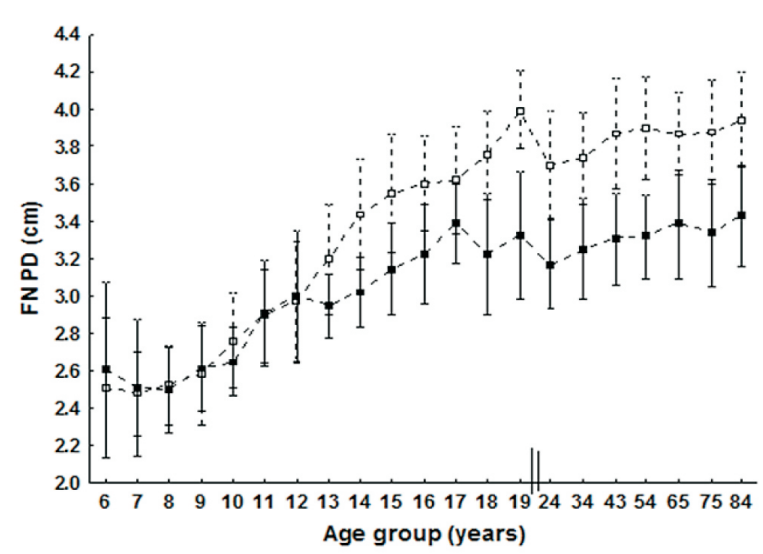

$\mathbf{E}$

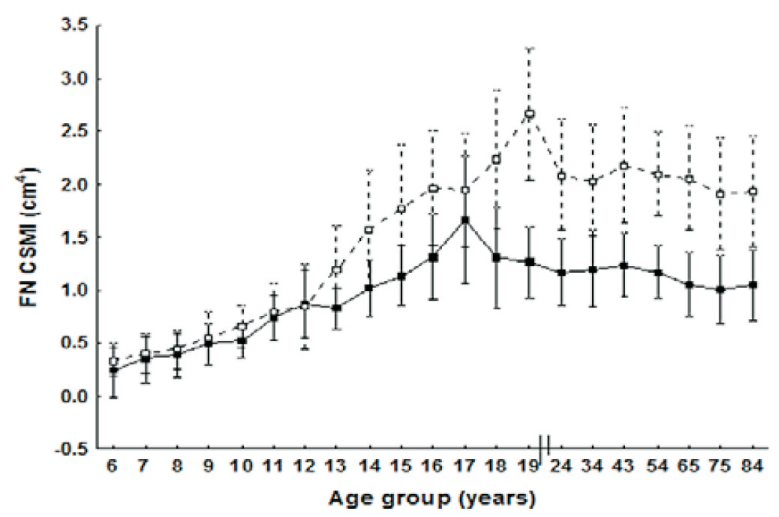

Girls
B

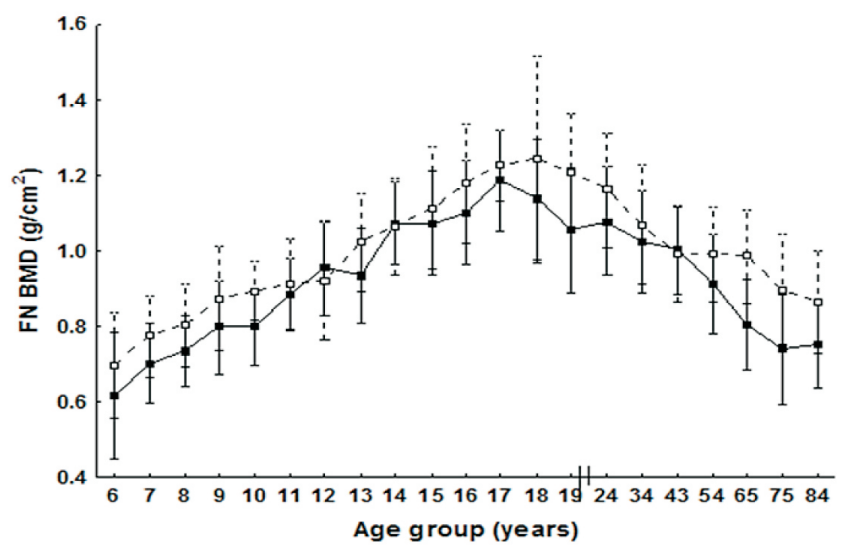

D
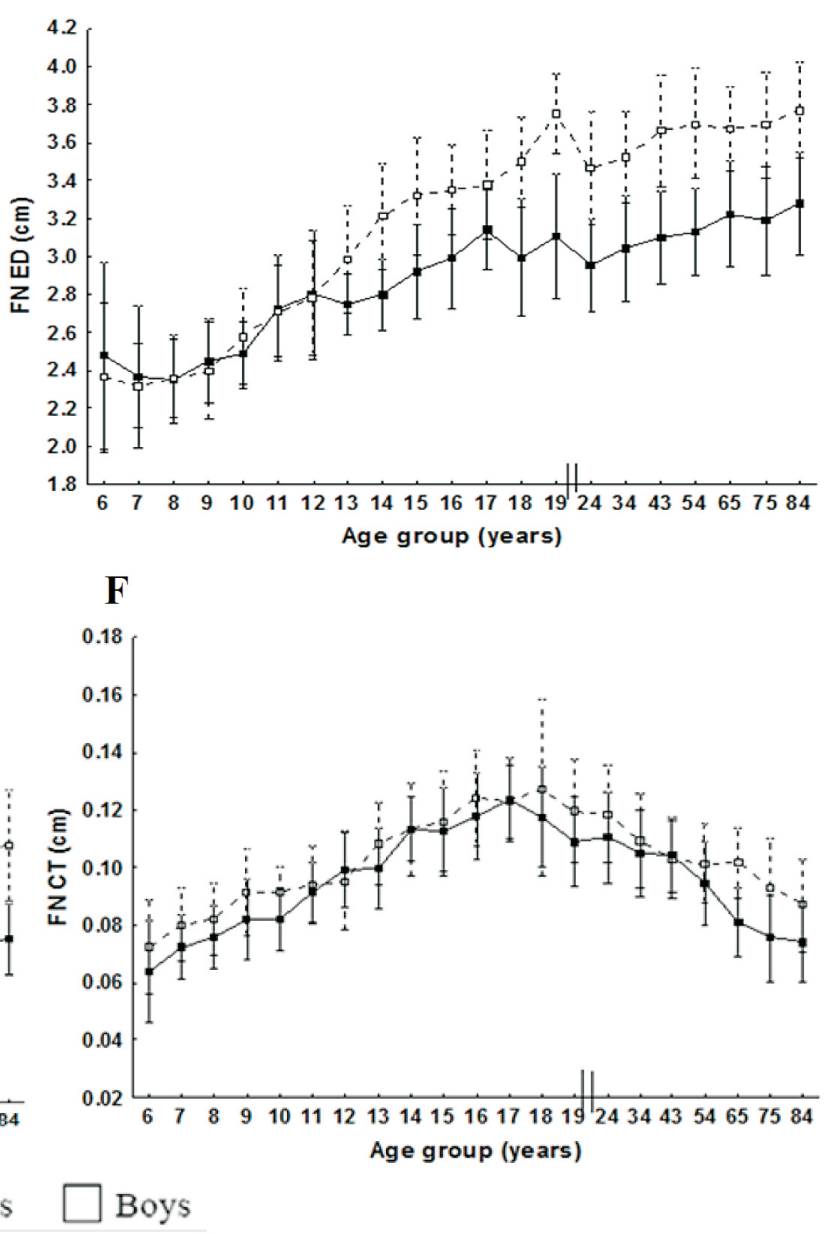

Figure 3: Age and sex specific changes in femoral neck geometric adaptions. A- FN BMC, B- FN BMD, C- FN periosteal diameter (PD), D- FN endosteal diameter (ED), E- FN cross-sectional moment of inertia (CSMI), F- cortical thisckness (CT). Data presented as mean (SD) 
Table 3: Annual Changes in FN Geometry and Mechanical Estimates. * $p<0.05$

\begin{tabular}{lcccc}
\hline Variable & $\begin{array}{c}\text { Mean relative annual changes } \\
\text { before peak point }(\%)\end{array}$ & $\begin{array}{c}\text { Mean relative annual changes } \\
\text { after peak point (\%) }\end{array}$ \\
\cline { 2 - 5 } & Boys & Girls & Men & Women \\
\hline FN BMC & $+10.2^{*}$ & $+9.2^{*}$ & $-0.4^{*}$ & $-0.6^{*}$ \\
FN BMD & $+4.9^{*}$ & $+5.8^{*}$ & -0.4 & $-0.6^{*}$ \\
FN PD & $+4.7^{*}$ & $+3.1^{*}$ & $+0.1^{*}$ & +0.1 \\
FN ED & $+4.7^{*}$ & $+2.8^{*}$ & $+0.2^{*}$ & +0.1 \\
FN CT & $+5.0^{*}$ & $+6.0^{*}$ & $-0.5^{*}$ & $-0.7 *$ \\
FN CSA & $+10.0 *$ & $+9.1^{*}$ & -0.3 & $-0.6 *$ \\
FN CSMI & $+21.1^{*}$ & $+15.5^{*}$ & 0.0 & -0.3 \\
FN Z & $+15.9^{*}$ & $+12.0^{*}$ & -0.1 & $-0.3^{*}$ \\
\hline
\end{tabular}

The finding that sex discrepancies in the development of bone mass and FN geometry adaptations during puberty continues into adolescence and adulthood, could explain the higher risk of FN fractures in women compared to men.

In conclusion, age related loss of FN bone mass is partly compensated by the increase in FN diameters and biomechanical indices. These structural and biomechanical adaptations are important to maintain FN bone strength. Preservation of FN bone strength in elderly is more obvious in men than in women. Findings of this study were published in Calcified Tissue International in 2012 (18).

\section{Associations of anthropometry and body composition on FN geometry in children and adolescents:}

Anthropometry and body composition are important factors associated with bone strength. The study was aimed to evaluate the relationships of anthropometry and body composition on FN geometric indices in children and adolescents.

For this study, 256 girls and 303 boys aged 6 to 14 participated and the adolescent group comprised of 326 girls and 319 boys aged 10 to 19.0 years. FN geometric indices and biomechanical properties evaluated by HSA at the narrowest FN were considered as dependent variables. These five FN development predictors; body weight, body height, BMI, TBLM and Log-transformed TBFM, were considered as the independent variables. Pearson correlation (r) and advanced, step-forward multiple regression analysis were applied.

\section{Results}

Anthropometry and body composition measures of girls and boys in both pre-pubertal and adolescent groups show that boys are significantly taller and heavier with significantly higher TBLM and lower TBFM compared to girls of similar BMI. In pre-pubertal girls and boys, height showed the greatest independent positive association with all FN geometric indices whereas in adolescents, TBLM showed the greatest independent positive association with all FN geometric indices, especially in boys. TBFM showed negative associations with FN bone mass and geometry in pre-pubertal children and adolescent boys.

In conclusion height in pre-pubertal children and TBLM in adolescents showed the greatest independent positive associations with FN geometry -especially in boys. Fat mass showed negative association with FN geometry and bone mass in pre-pubertal children and adolescent boys.

As childhood obesity is an increasing problem, findings of the current study showed an increasing interest in both the clinical and the research situations when trying to prevent obesity associated growth disturbances during growth. Relative independent positive association of lean mass and 
negative association of fat mass on FN geometry in children and adolescents need to be investigated in future longitudinal studies. The findings of this study were presented as abstracts in the $25^{\text {th }}$ annual scientific meeting of International Society for Clinical Densitometry (ISCD 2019) in Kuala Lumpur Malaysia $(19,20,21)$.

\section{Overall Conclusions}

School-based moderately intense PE exercises have direct beneficial effects on increasing the bone strength in LS in pre pubertal children. The age and sex specific total and regional soft tissues, bone mass in the different skeletal sites were observed, with the appendicular skeleton having an extended period of increased BMC into adulthood compared to the axial skeleton. Age related loss of FN bone mass is partially compensated by changing the FN bone geometry. Height in children and TBLM in adolescents show the greatest independent positive associations with FN geometry, especially in adolescent boys. In addition, the increase of the muscle mass and decrease of the fat mass seems to have beneficial effects on developing better femoral neck geometry, especially in boys. Despite the fact that this study was conducted in Sweden with Swedish children and adolescents, applying this message in Sri Lanka and allowing the Sri Lankan children to play in an enjoyable environment would be an important approach when aiming better musculoskeletal health.

\section{References}

1. Cooper C, Campion G, Melton LJ, Third Hip fractures in the elderly: a world-wide projection. Osteoporosis International: a journal established as result of cooperation between the European Foundation for Osteoporosis and the National Osteoporosis Foundation of the USA. 1992; 2(6): 285-9.

2. Faulkner KG, Wacker WK, Barden HS, Simonelli C, Burke $\mathrm{PK}$, Ragi S, et al. Femur strength index predicts hip fracture independent of bone density and hip axis length. Osteoporosis International: a journal established as result of cooperation between the European Foundation for Osteoporosis and the National Osteoporosis Foundation of the USA. 2006; 17(4): 593-9.
3. Beck TJ, Oreskovic TL, Stone KL, Ruff CB, Ensrud K, Nevitt MC, et al. Structural adaptation to changing skeletal load in the progression toward hip fragility: the study of osteoporotic fractures. Journal of bone and mineral research: the official journal of the American Society for Bone and Mineral Research, 2001; 16(6): 1108-19.

4. Felsenberg D, Boonen S. The bone quality framework: determinants of bone strength and their interrelationships, and implications for osteoporosis management. Clin Ther, 2005; 27(1): 1-11.

5. Hui SL, Slemenda CW, Johnston CC, Jr. The contribution of bone loss to postmenopausal osteoporosis. Osteoporosis International: a journal established as result of cooperation between the European Foundation for Osteoporosis and the National Osteoporosis Foundation of the USA. 1990; 1(1):30-4.

6. MacKelvie KJ, Khan KM, McKay HA. Is there a critical period for bone response to weight-bearing exercise in children and adolescents? a systematic review. British Journal of Sports Medicine, 2002; 36(4): 250-7; Discussion 7.

7. Gordon CM, Bachrach LK, Carpenter TO, Crabtree N, El-Hajj Fuleihan G, Kutilek S, et al. Dual energy X-ray absorptiometry interpretation and reporting in children and adolescents: the 2007 ISCD Pediatric Official Positions. Journal of Clinical Densitometry: the official journal of the International Society for Clinical Densitometry. 2008; 11(1): 43-58.

8. Beck TJ, Ruff CB, Warden KE, Scott WW, Jr., Rao GU. Predicting femoral neck strength from bone mineral data. A structural approach. Invest Radiol, 1990; 25(1): 6-18.

9. Beck TJ, Looker AC, Ruff CB, Sievanen H, Wahner HW. Structural trends in the aging femoral neck and proximal shaft: analysis of the Third National Health and Nutrition Examination Survey dual-energy X-ray absorptiometry data. Journal of Bone and Mineral Research: the official Journal of the American Society for Bone and Mineral Research. 2000; 15(12): 2297-304.

10. Valdimarsson O, Linden C, Johnell O, Gardsell P, Karlsson MK. Daily physical education in the school curriculum in prepubertal girls during 1 year is followed by an increase in bone mineral accrual and bone width data from the prospective controlled Malmo pediatric osteoporosis prevention study. Calcified Tissue International, 2006; 78(2): 65-71. 
11. Alwis G, Linden C, Ahlborg HG, Dencker M, Gardsell P, Karlsson MK. A 2-year school-based exercise programme in pre-pubertal boys induces skeletal benefits in lumbar spine. Acta Paediatr, 2008; 97(11): 1564-71.

12. Alwis G, Linden C, Stenevi-Lundgren S, Ahlborg HG, Besjakov J, Gardsell P, et al. A one-year exercise intervention program in pre-pubertal girls does not influence hip structure. BMC Musculoskeletal Disorders, 2008; 9:9.

13. Alwis G, Linden C, Stenevi-Lundgren S, Ahlborg HG, Dencker M, Besjakov J, et al. A school-curriculum-based exercise intervention program for two years in pre-pubertal girls does not influence hip structure. Dyn Med, 2008; 7: 8 .

14. Linden C, Alwis G, Ahlborg H, Gardsell P, Valdimarsson $\mathrm{O}$, Stenevi-Lundgren S, et al. Exercise, bone mass and bone size in prepubertal boys: one-year data from the pediatric osteoporosis prevention study. Scandinavian journal of medicine \& science in sports. 2007; 17(4): 340-7.

15. Nordstrom P, Neovius M, Nordstrom A. Early and rapid bone mineral density loss of the proximal femur in men. JClin Endocrinol Metab, 2007; 92(5): 1902-8.

16. Faulkner RA, Bailey DA, Drinkwater DT, McKay HA, Arnold C, Wilkinson AA. Bone densitometry in Canadian children 8-17 years of Age. Calcified Tissue International, 1996; 59(5): 344-51.
17. Alwis G, Rosengren B, Stenevi-Lundgren S, Duppe H, Sernbo I, Karlsson MK. Normative dual energy X-ray absorptiometry data in Swedish children and adolescents. Acta Paediatr, 2010; 99(7): 1091-9.

18. Alwis G, Karlsson C, Stenevi-Lundgren S, Rosengren BE, Karlsson MK. Femoral neck bone strength estimated by hip structural analysis (HSA) in Swedish Caucasians aged 6-90 years. Calcified Tissue International, 2012; 90(3): 174-85.

19. Alwis G, Lenora J, Karlsson MK. Associations of Anthropometry and Body Composition on Femoral Neck Geometry in Swedish Adolescents, (Oral presentation) (BT 04) at $25^{\text {th }}$ ISCD, Kaula Lumpur, Malaysia, $22^{\text {nd }}$ March 2019.

20. Alwis G, Lenora J, Karlsson MK. Associations of Anthropometry and Body Composition on Femoral Neck Geometry in Pre-pubertal Girls, (Poster presentation) (MM 02) at $25^{\text {th }}$ ISCD, Kaula Lumpur, Malaysia, $20^{\text {th }}-23^{\text {rd }}$ March 2019.

21. Alwis G, Lenora J, Karlsson MK. Associations of Anthropometry and Body Composition on Femoral Neck Geometry in Pre-pubertal Boys, (Poster presentation) (MM 03) at $25^{\text {th }}$ ISCD, Kaula Lumpur, Malaysia, $20^{\text {th }}-23^{\text {rd }}$ March 2019. 